\title{
Phenotypic and Genotypic Variability in a Set of Sesame (Sesamum indicum L.) Genotypes
}

\author{
Ismail M. Bedawy ${ }^{\#}$ and Naheif E. Mohamed \\ Agronomy Department, Faculty of Agriculture, Sohag University, Sohag, Egypt.
}

$\mathbf{T}$

OTALLY, 86 sesame (Sesamum indicum L.) genotypes were used for the study of-phenotypic and genotypic variability in summer seasons of 2015 and 2016. They were differed significantly values for all studied traits in both seasons. The genotypes mean ranged widely around the general mean in the studied traits. 67.4, $40.7,46.5$ and $48.8 \%$ of the genotypes were raised than the average for plant height $(159.05 \mathrm{~cm})$, capsule length $(2.56 \mathrm{~cm})$, thousand seed weight $(3.65 \mathrm{~g})$ and seed yield per plant $(14.38 \mathrm{~g})$. While $51.2 \%$ of genotypes were higher than the average of first capsule height, length of fruiting zone and capsules number per plant in the first season. The higher phenotypic values than overall mean observed as 53.5, 51.2, 52.3, 54.7, 40.7, 48.8 and $48.8 \%$ for the plant height, the first capsule height, the fruiting zone length, the capsule number per plant, the capsule length, the thousand seed weight and the seed yield per plant in the second year, respectively. Also, high values $(>20 \%)$ of phenotypic coefficient variations (PCV) and genotypic coefficients of variations (GCV) were recorded in the first capsule height in both seasons. However, moderate values of genetic advance (GA) were observed for the plant height (18.49) in both seasons and (17.52 \& 16.94) for the fruiting zone length in the first and second seasons coupled with high heritability. Higher heritability coupled with high genetic advance estimates $(>20)$ were recorded for the first capsule height (26.05 and 25.85) and the capsules number per plant (22.55 and 26.11) in both seasons. This is indicates that these traits controlled by additive gene action and mostly which is very useful for selection efficiency.

Keywords: Sesame (Sesamum indicum L.), Genetic variability, Phenotypic coefficient of variation (PCV), Genotypic coefficient of variation (GCV), Heritability.

\section{Introduction}

Sesame (Sesamum indicum L.) is one of the oldest crops cultivated and widely grown in tropical and subtropical zones from $40^{\circ} \mathrm{N}$ to $40^{\circ} \mathrm{S}$ latitude. The cultivated sesame area mostly occurs in the developing countries such as India, Myanmar, China, Sudan and Nigeria (Hassan, 2013 and Hamza \& Abd El-Salam, 2015).

In Egypt, there is a big gap between the total production and consumption of edible oils, whereas the self-sufficient is about $10 \%$ and $90 \%$ imported. Similarly, its plant is characterized by low nitrogen content and water requirements, and seeds are rich in oil seed (50-60\%) and some of the essential amino acids such as linoleic acid, which is a source of vitamin $\mathrm{E}$, calcium and phosphorous minerals (Weiss, 2000 and Hamza \& Abd El-Salam, 2015). In addition to them, it is being reported that this plant could be grown at the reclaimed soils (Boureima et al., 2016). Despite of all these advantages, the local production of sesame is 45 thousand tonnes, harvested from a small cultivated area of 32 thousand ha (FAOSTAT, 2016). The limited area is due to the low yielding capacity of the plant compared to other crop plants, its susceptibility to diseases, seed shattering, indeterminate growth habit and asynchronous capsule ripening (Ashri, 1998 and Yol \& Uzun, 2012). To minimize the edible oil gap, Egypt needs to create a national program for

\#Corresponding author email: ismail_bedawy@yahoo.com

DOI: 10.21608 /agro.2018.4719.1108

C2018 National Information and Documentation Center (NIDOC) 
expanding the cultivated sesame area, especially in the reclaimed desert lands. In parallel with a strong breeding program effective for developed high yielding genotypes under the abiotic stress in the reclaimed soil. Herein, Boureima et al. (2016) reported that genetic and breeding improvement efforts in the sesame have been limited, making the results of such efforts slow to emerge. Effective selection criteria and additional genetic information for breeders are insufficient. So would be used effectiveness selection methods in which associated criteria with the sesame yield. Wide information on phenotypic and genotypic variability would be available for a plant breeder. Hika et al. (2015) stated that using the germplasm in the improvement program is considered a great opportunity for the genotype selection of high yielding productivity. The higher PCV rather than the GCV indicates that trait expression is highly affected by environmental effects. Broad sense heritability refers to the percentage of genetic sharing for the trait expressions. Sabiel et al. (2015) found the highest GCV for seed yield and high the heritability for days to flowering $(>85 \%)$ in their studied genetic variation of sesame. Spandana et al. (2011) found the high PCV and GCV for the seed yield per plant. Also, highly values of the PCV and the GCV were reported by Gidey et al. (2012) for the first capsule height, the seed yield, the number of seeds per capsule and the day to $50 \%$ flowering. They also reported the high heritability value for the first capsule height and the days to $50 \%$ flowering. Similarly, Mahdy et al. (2005 a and b) were observed high heritability for the plant height, the first capsule height, the days to $50 \%$ blooming, the capsules per plant, the capsule length, the seed yield per plant and the thousand seed weight.

The broad sense heritability estimated alone is not reliable, because these estimations values are might be affected by the environmental and plant material changes. Using high broad sense heritability $\left(\mathrm{H}_{\mathrm{b}}\right)$ coupled with higher genetic advance (GA) is more preferred and helpful in prediction gain under selection due to the additive gene action, reported by El Soury et al. (2016) and Patil \& Lokesha (2018). Also, Abate et al. (2015) observed moderate to high genetic advance in the number capsules, biomass and harvest index and the thousand seed weight coupled with the moderate heritability and these traits were controlled by the additive and non-additive genes.

The current investigation aimed to: (1) Study the phenotypic and genotypic variability in the seed yield and related traits of the used sesame genotypes, (2) Identify the traits which highly expressed by genetic rather than the environmental effects and (3) Observe the traits which controlled by the additive gene action for using efficiently selection methods.

\section{Materials and Methods}

A population of 6 sesame varieties and 80 sesame lines were used in this study. (Developed in the previous breeding program for high yielding and resistant to wilt diseases (Mahdy et al., $2005 \mathrm{a}$ and b). This experiment was carried out at the Research Farm of Faculty Agric., Sohag University at El-Kawthar during the summer seasons of 2015 and 2016 to study the phenotypic and genotypic variability. A randomized complete block design (RCBD) with 3 replicates was used. Seeds were sown in rows of $4 \mathrm{~m}$ long, $60 \mathrm{~cm}$ apart and $20 \mathrm{~cm}$ between hills within rows (two rows for one genotype). At harvest 20 guarded plants were chosen randomly from each genotype, then the following traits were recorded as average: (1) The plant height $(\mathrm{cm}),(2)$ The first capsule height $(\mathrm{cm}),(3)$ The fruiting zone length $(\mathrm{cm}),(4)$ The capsules number per plant, (5) The capsule length $(\mathrm{cm}),(6)$ The thousand seed weight $(\mathrm{g})$ and (7) The seed yield per plant (g).

The recorded data were statistically analyzed in SAS statistical software (SAS ver. 9.2, SAS Institute, 2008). The revised LSD at $5 \%$ and $1 \%$ significant levels were calculated according to Petersen (1985), for comparing the genotypes mean values of the studied traits. In addition, the parameters for the phenotypic and genotypic variability were calculated according to Burton (1952) and Al-Jibouri et al. (1958) as follow:

\section{1- Genotypic variance $(\mathrm{Vg})=(\mathrm{MSg}-\mathrm{MSe}) / \mathrm{r}$}

where, MSg is the genotype mean square, MSe is the mean square of experimental error and $r$ is the number of replicates.

2- Phenotypic variance $(\mathrm{Vp})=\mathrm{Vg}+\mathrm{MSe}$

3- Phenotypic coefficient of variation

$$
(\mathrm{PCV})=\sqrt{\frac{V p}{\bar{x}}} * 100
$$

4- Genotypic coefficient of variation 


$$
(\mathrm{GCV})=\sqrt{\frac{V g}{\bar{x}}} * 100
$$

5- Broad sense heritability $(\mathrm{H})=(\mathrm{Vg} / \mathrm{Vp}) * 100$

6- Genetic Advance $(\mathrm{GA})=\mathrm{k}^{*} \mathrm{H} * \sqrt{V p}$

where, $\mathrm{k}$ is the intensity of selection $(\mathrm{k}=2.06$ at $5 \%$ selection intensity).

7- Genetic advance over mean $(\mathrm{GAM})=(\mathrm{GA} /$ $\bar{x})^{*} 100$

\section{Results and Discussion}

\section{Phenotypic parameters}

The sesame genotypes differed significantly at $5 \%$ and $1 \%$ levels in all studied traits in both seasons. Results were indicated that genetic variability is existing among-sesame genotypes in terms of investigated traits (Table 1). So, this variation could be a good chance for improving these traits under selection process. These results are in agreement with those obtained by Saha et al. (2012), Begum et al. (2017), Aye et al. (2018) and Patil \& Lokesha (2018). On the other hand, the phenotypic values (Tables 2 $\& 3$ and Fig. 1 \& 2) were also ranged widely around the mean values and represented 67.4, 40.7, 46.5 and $48.8 \%$ of the genotypes above the average for the plant height, the capsule length, the thousand seed weight and the seed yield per plant, while $51.2 \%$ of genotypes were higher than the average for the both of first capsule height, the length of fruiting zoon and the capsules number per plant in the first season, respectively. The higher phenotypic values of the genotypes than overall mean were 53.5, 51.2, $52.3,54.7$, and $40.7 \%$ for the plant height, the first capsule height, the fruiting zoon length, the capsules number per plant, the capsule length and $48.8 \%$ for the both of thousand seed weight and the seed yield per plant in the second season, respectively. The maximum values of studied traits were observed in the genotypes number $3(175 \mathrm{~cm}), 9(86 \mathrm{~cm}), 41$ (123.67 cm), 40 (161.33capsule), $86(3.16 \mathrm{~cm}), 34$ $(4.7 \mathrm{~g})$ and $6(18.31 \mathrm{~g})$ for the plant height, the first capsule height, the length of fruiting zoon and the capsules number per plant, the capsule length, the thousand seed weight and the seed yield per plant in the first season, respectively. Furthermore, the genotype number 66 exhibited values above the mean in all studied traits (Tables 2 and 3 ).

Regarding to the second season, the highest values $(178.33 \mathrm{~cm}, 86 \mathrm{~cm}, 124 \mathrm{~cm}, 163.67$ capsules,
$3.17 \mathrm{~cm}, 4.86 \mathrm{~g}$ and $16.64 \mathrm{~g}$ ) were obtained from the genotypes number $2,9,44,70,86,34$ and 6 for the plant height, the first capsule height, the fruiting zoon length, the capsules number per plant, the capsule length, the thousand seed weight and the seed yield per plant in the second season, respectively. While the genotypes of number 54 and 66 were exhibited the higher values in all studied characters. These results as similar to those obtained by Hika et al. (2015). They were found highly significant differences intra 64 populations for seed yield and its components and stated that 52 and $40.63 \%$ of the populations were higher than the mean of thousand seed weight and the seed yield, respectively.

\section{Genotypic parameters}

The phenotypic and genotypic variability plays an important role in sesame breeding program across hybridization and selection (Begum \& Dasgupta, 2014; Begum et al., 2017 and Aye et al., 2018). The presented data in Tablel and Fig. 3 show that the PCV and GCV are close together for most studied traits in both seasons, indicating the minimum environmental effects on the genetic expression of studied traits, herein the selection based on phenotypic values will not be so difficult. These results confirmed by Patil \& Lokesha (2018). They reported that the PCV was higher than the GCV for the yield and its attributes with low difference interpreted environmental effect which was low and the genetic factors controlling variability in these traits. Results in the Table 1 showed high values of the PCV and the GCV (>20\%) were recorded in the first capsule height $(25.64$ and $25.45 \mathrm{~cm})$ and $(25.52$ and $25.31 \mathrm{~cm})$ in the first and second seasons, respectively. The fruiting zone length, the capsules number per plant, the thousand seed weight and the seed yield per plant were moderate (10-20\%) values of the PCV and the GCV in both seasons. In the same line, Hika et al. (2015) found moderate values of the PCV and the GCV for the seed yield (18.31 and 18.30\%), low values for the plant height (6.27 and 6.14) and the thousand seed weight (11.56 and 7.57\%). Similar results were obtained by Khan et al. (2007). They concluded that maximum percentage of the GCV and the PCV (33.58\% and 34.26\%) were observed for the seed yield per $\mathrm{m}^{2}$ followed by $26.80 \%$ and $29.17 \%$ for the seed yield per plant. On the other hand, low values of the PCV and the GCV $(<10 \%)$ were found with the plant height and the capsule length in both seasons (Table 1). This means that 
these traits are highly affected by environmental conditions. These results are in line with those obtained by Shekhawat et al. (2013), Bharathi et al. (2014), Aristya et al. (2017) and Saxena $\&$ Bisen (2017). Broad sense heritability is significant to the breeding programs, in regards to the percentage of genetic expressing the character phenotype. In the current study, Heritability in broad sense estimations (Table 1) were high for all studied traits and ranged from $87.58 \%$ for capsules number per plant to $98.5 \%$ for the first capsule height and from $89.24 \%$ for the fruiting zone length to $98.43 \%$ for the first capsule height in the first and second seasons, respectively. This indicates that small contribution of the environment in the phenotype expression. These results are in agreement with those obtained by Hika et al. (2015). They found that broad sense heritability values were more than $80 \%$ for all traits except the thousand seed weight (45.13\%). The highest heritability percentages $(91.40,88.70$ and $86.20 \%$ ) were recorded for the seed yield per plant, the plant height and the capsules number per plant, respectively (Begum et al., 2017). Also, Khan et al. (2007) observed the low difference among the GCV and the PCV and coupled with high broad sense heritability for all the characters in their study. They added that selection would be effective for the yield and its components.

TABLE 1. Analysis of variance, means, ranges, phenotypic (PCV), genotypic (GCV) coefficients of variation and heritability in a broad sense $(\mathrm{H})$ for examined traits.

\begin{tabular}{|c|c|c|c|c|c|c|c|c|}
\hline Item & & $\begin{array}{l}\text { Plant height } \\
\text { (cm) }\end{array}$ & $\begin{array}{c}\text { First capsule } \\
\text { height } \\
(\mathrm{cm})\end{array}$ & $\begin{array}{l}\text { Fruiting } \\
\text { zone length } \\
\text { (cm) }\end{array}$ & $\begin{array}{c}\text { Capsules per } \\
\text { plant }\end{array}$ & $\begin{array}{c}\text { Capsule } \\
\text { length } \\
(\mathrm{cm})\end{array}$ & $\begin{array}{c}\text { Thousand } \\
\text { seed weight } \\
\text { (g) }\end{array}$ & $\begin{array}{l}\text { Seed yield/ } \\
\text { plant } \\
\text { (g) }\end{array}$ \\
\hline S.O.V & DF & \multicolumn{7}{|c|}{ Mean square at 2015 season } \\
\hline Replication & 2 & $33.878^{*}$ & 5.236 & 0.457 & 59.468 & 0.0015 & 0.0382 & 0.239 \\
\hline Genotypes & 85 & $269.37 * *$ & $494.33 * *$ & $254.18^{* *}$ & $466.74 * *$ & $0.0518^{* *}$ & $0.262 * *$ & $9.365^{* *}$ \\
\hline Error & 170 & 7.37 & 3.746 & 10.14 & 30.884 & 0.00179 & 0.0113 & 0.532 \\
\hline Mean & & 159.05 & 61.54 & 97.51 & 137.05 & 2.56 & 3.65 & 14.38 \\
\hline Range & & $128-175$ & $28.67-86$ & $\begin{array}{l}80.67- \\
123.67\end{array}$ & $107.3-161.33$ & $2.34-3.16$ & $2.98-4.7$ & $10.98-18.31$ \\
\hline PCV & & 7.40 & 25.64 & 11.79 & 11.51 & 6.40 & 10.12 & 15.46 \\
\hline GCV & & 7.20 & 25.45 & 11.33 & 10.77 & 6.18 & 9.69 & 14.61 \\
\hline $\mathrm{H}$ & & 94.67 & 98.50 & 92.33 & 87.59 & 93.32 & 91.73 & 89.25 \\
\hline GA & & 18.49 & 26.05 & 17.52 & 22.55 & 0.25 & 0.56 & 3.25 \\
\hline \multirow{2}{*}{ GAM } & & 11.62 & 42.32 & 17.97 & 16.45 & 9.87 & 15.30 & 22.61 \\
\hline & & Moderate & High & Moderate & Moderate & Low & Moderate & High \\
\hline S.O.V & DF & \multicolumn{7}{|c|}{ Mean square at 2016 season } \\
\hline Replication & 2 & 2.794 & 5.306 & 0.399 & 14.84 & 0.0012 & 0.00108 & 0.473 \\
\hline Genotypes & 85 & $283.637 * *$ & $487.61 * *$ & $254.022 * *$ & $559.35^{* *}$ & $0.0526^{* *}$ & $0.25031^{* *}$ & $10.99 * *$ \\
\hline Error & 170 & 11.445 & 3.86 & 14.442 & 21.045 & 0.00102 & 0.01111 & 0.551 \\
\hline Mean & & 160.50 & 61.44 & 99.07 & 138.05 & 2.57 & 3.68 & 14.76 \\
\hline Range & & $128-178.33$ & $28.67-86$ & $80-124$ & $103.33-163.67$ & $2.37-3.17$ & $2.99-4.86$ & $11.12-18.94$ \\
\hline PCV & & 7.57 & 25.52 & 11.70 & 12.34 & 6.37 & 9.81 & 16.27 \\
\hline GCV & & 7.27 & 25.31 & 11.05 & 11.88 & 6.25 & 9.39 & 15.48 \\
\hline $\mathrm{H}$ & & 92.24 & 98.43 & 89.24 & 92.75 & 96.20 & 91.50 & 90.45 \\
\hline GA & & 18.49 & 25.85 & 16.94 & 26.11 & 0.26 & 0.54 & 3.57 \\
\hline \multirow{2}{*}{ GAM } & & 11.52 & 42.08 & 17.10 & 18.91 & 10.21 & 14.79 & 24.19 \\
\hline & & Moderate & High & Moderate & Moderate & Moderate & Moderate & High \\
\hline
\end{tabular}

DF: Degrees of freedom; *** Significant and highly significant, respectively; GA: Genetic advance and GAM: Genetic advance over mean. 
TABLE 2. Means of studied traits for the 86 sesame genotypes in 2015 season.

\begin{tabular}{|c|c|c|c|c|c|c|c|c|}
\hline $\begin{array}{l}\text { Genotype } \\
\text { No. }\end{array}$ & Code & $\begin{array}{c}\text { Plant } \\
\text { height } \\
(\mathrm{cm})\end{array}$ & $\begin{array}{c}\text { First } \\
\text { capsule } \\
\text { height } \\
(\mathrm{cm})\end{array}$ & $\begin{array}{c}\text { Fruiting } \\
\text { zone } \\
\text { length } \\
\text { (cm) }\end{array}$ & $\begin{array}{c}\text { Number } \\
\text { of capsules } \\
\text { per plant }\end{array}$ & $\begin{array}{c}\text { Capsule } \\
\text { length } \\
(\mathrm{cm})\end{array}$ & $\begin{array}{l}\text { Thousand } \\
\text { seed weight } \\
\text { (g) }\end{array}$ & $\begin{array}{c}\text { Seed } \\
\text { yield } \\
(\mathrm{g})\end{array}$ \\
\hline 1 & $1 \_1 \_1$ & 171.83 & 67.67 & 104.17 & 139.67 & 2.37 & 3.53 & 13.24 \\
\hline 2 & $1 \_1 \_2$ & 169.00 & 69.67 & 99.33 & 139.67 & 2.48 & 3.51 & 13.99 \\
\hline 3 & 5_1_1 & 175.33 & 76.67 & 98.67 & 142.00 & 2.46 & 3.47 & 16.47 \\
\hline 4 & $5 \_1 \_2$ & 170.67 & 77.00 & 93.67 & 141.00 & 2.65 & 3.40 & 16.58 \\
\hline 5 & 9_1_1 & 173.33 & 76.33 & 97.00 & 154.67 & 2.63 & 3.71 & 16.61 \\
\hline 6 & 9_1_2 & 171.33 & 75.00 & 96.33 & 160.33 & 2.57 & 3.85 & 18.31 \\
\hline 7 & 9_2_1 & 167.33 & 79.67 & 87.67 & 139.33 & 2.50 & 4.25 & 13.59 \\
\hline 8 & 9_2_2 & 168.00 & 80.00 & 88.00 & 136.00 & 2.51 & 3.95 & 14.22 \\
\hline 9 & 10_1 & 167.33 & 86.00 & 81.33 & 134.67 & 2.52 & 3.63 & 15.41 \\
\hline 10 & $10 \_2$ & 166.33 & 84.00 & 82.33 & 132.67 & 2.57 & 3.93 & 15.16 \\
\hline 11 & 11_2_1 & 165.33 & 73.67 & 91.67 & 159.67 & 2.45 & 3.64 & 16.69 \\
\hline 12 & $11 \_2 \_2$ & 162.33 & 75.00 & 87.33 & 157.00 & 2.44 & 4.14 & 16.75 \\
\hline 13 & 266_1 & 157.67 & 64.33 & 93.33 & 145.33 & 2.35 & 3.80 & 14.86 \\
\hline 14 & $266 \_2$ & 155.33 & 62.67 & 92.67 & 143.33 & 2.38 & 3.96 & 14.86 \\
\hline 15 & 216_1 & 158.00 & 61.67 & 96.33 & 142.33 & 2.46 & 3.79 & 14.62 \\
\hline 16 & $216 \_2$ & 158.33 & 64.33 & 94.00 & 142.33 & 2.39 & 3.60 & 15.37 \\
\hline 17 & $257 \_1$ & 152.67 & 56.67 & 96.00 & 133.33 & 2.38 & 3.40 & 11.23 \\
\hline 18 & 257_2 & 160.33 & 58.67 & 101.67 & 131.33 & 2.61 & 3.70 & 12.59 \\
\hline 19 & $44 \_1$ & 169.00 & 64.67 & 104.33 & 124.00 & 2.50 & 3.30 & 12.65 \\
\hline 20 & 44_2 & 170.33 & 70.67 & 99.67 & 128.00 & 2.43 & 3.16 & 12.44 \\
\hline 21 & 34_2_1 & 170.67 & 72.33 & 98.33 & 138.33 & 2.63 & 3.16 & 15.91 \\
\hline 22 & $34 \_2 \_2$ & 173.00 & 70.67 & 102.33 & 137.67 & 2.68 & 3.06 & 15.70 \\
\hline 23 & 59_1_1 & 154.67 & 46.00 & 108.67 & 137.00 & 2.57 & 4.05 & 14.43 \\
\hline 24 & 59_1_2 & 158.00 & 52.00 & 106.00 & 137.00 & 2.63 & 3.43 & 13.69 \\
\hline 25 & 110_12_1 & 154.67 & 60.67 & 94.00 & 114.00 & 2.60 & 3.49 & 12.48 \\
\hline 26 & 110_12_2 & 152.67 & 59.00 & 93.67 & 136.33 & 2.77 & 3.54 & 12.22 \\
\hline 27 & 58_3_1 & 145.67 & 60.67 & 85.00 & 135.67 & 2.53 & 3.02 & 13.49 \\
\hline 28 & $58 \_3 \_2$ & 151.67 & 56.33 & 95.33 & 135.67 & 2.68 & 3.41 & 14.41 \\
\hline 29 & $58 \_1$ & 158.33 & 55.67 & 102.67 & 146.00 & 2.75 & 3.87 & 17.37 \\
\hline 30 & $58 \_2$ & 157.67 & 60.00 & 97.67 & 147.00 & 2.68 & 3.90 & 16.99 \\
\hline 31 & 167_1_1 & 153.00 & 40.67 & 112.33 & 120.33 & 2.48 & 3.52 & 12.13 \\
\hline 32 & 167_1_2 & 160.00 & 43.00 & 117.00 & 121.00 & 2.37 & 3.72 & 12.59 \\
\hline 33 & 148_1 & 148.67 & 48.33 & 100.33 & 140.33 & 2.53 & 4.02 & 16.93 \\
\hline 34 & $148 \_2$ & 154.33 & 48.67 & 105.67 & 143.67 & 2.69 & 4.70 & 16.92 \\
\hline 35 & $65 \_1$ & 141.33 & 38.67 & 102.67 & 117.00 & 2.61 & 4.14 & 12.68 \\
\hline 36 & $65 \_2$ & 149.00 & 45.00 & 104.00 & 117.33 & 2.48 & 3.70 & 13.23 \\
\hline 37 & $76 \_1$ & 166.33 & 79.00 & 87.33 & 139.33 & 2.49 & 3.77 & 14.68 \\
\hline 38 & $76 \_2$ & 165.33 & 83.67 & 81.67 & 138.33 & 2.43 & 3.63 & 14.79 \\
\hline 39 & $1 \_1$ & 155.00 & 54.67 & 100.33 & 155.67 & 2.55 & 2.99 & 16.55 \\
\hline 40 & $1 \_2$ & 157.67 & 53.00 & 104.67 & 161.33 & 2.54 & 3.28 & 17.24 \\
\hline 41 & 2_4_1 & 161.67 & 38.00 & 123.67 & 142.67 & 2.47 & 3.35 & 15.24 \\
\hline 42 & 2_4_2 & 159.67 & 44.33 & 115.33 & 143.33 & 2.49 & 3.47 & 12.21 \\
\hline 43 & 3_1 & 172.67 & 53.67 & 119.00 & 137.00 & 2.49 & 3.68 & 14.14 \\
\hline 44 & 32 & 168.00 & 46.67 & 121.33 & 140.67 & 2.47 & 3.82 & 13.52 \\
\hline
\end{tabular}


TABLE 2. Cont.

\begin{tabular}{|c|c|c|c|c|c|c|c|c|}
\hline $\begin{array}{l}\text { Genotype } \\
\text { No. }\end{array}$ & Code & $\begin{array}{c}\text { Plant } \\
\text { height } \\
(\mathrm{cm})\end{array}$ & $\begin{array}{c}\text { First } \\
\text { capsule } \\
\text { height } \\
(\mathrm{cm})\end{array}$ & $\begin{array}{c}\text { Fruiting } \\
\text { zone } \\
\text { length } \\
(\mathrm{cm})\end{array}$ & $\begin{array}{l}\text { Number } \\
\text { of capsules } \\
\text { per plant }\end{array}$ & $\begin{array}{c}\text { Capsule } \\
\text { length } \\
(\mathrm{cm})\end{array}$ & $\begin{array}{l}\text { Thousand } \\
\text { seed } \\
\text { weight } \\
\text { (g) }\end{array}$ & $\begin{array}{c}\text { Seed } \\
\text { yield } \\
(\mathrm{g})\end{array}$ \\
\hline 45 & $58 \_1$ & 163.00 & 75.33 & 87.67 & 142.00 & 2.53 & 3.57 & 14.30 \\
\hline 46 & $58 \_2$ & 162.33 & 71.33 & 91.00 & 145.67 & 2.54 & 3.39 & 13.93 \\
\hline 47 & 125_1 & 149.67 & 39.67 & 110.00 & 125.00 & 2.53 & 3.41 & 11.56 \\
\hline 48 & $125 \_2$ & 153.33 & 45.33 & 108.00 & 124.33 & 2.52 & 3.53 & 12.13 \\
\hline 49 & $133 \_1$ & 168.00 & 82.00 & 86.00 & 151.00 & 2.41 & 3.19 & 16.96 \\
\hline 50 & $133 \_2$ & 164.33 & 81.33 & 83.00 & 152.67 & 2.49 & 3.40 & 15.96 \\
\hline 51 & 2_1_1 & 159.33 & 59.67 & 99.67 & 129.67 & 2.46 & 3.33 & 10.98 \\
\hline 52 & 2_1_2 & 161.00 & 60.33 & 100.67 & 128.33 & 2.39 & 3.52 & 13.00 \\
\hline 53 & 11_1_1 & 160.00 & 68.00 & 92.00 & 146.33 & 2.56 & 3.92 & 15.93 \\
\hline 54 & 11_1_2 & 164.67 & 67.33 & 97.33 & 148.00 & 2.60 & 3.79 & 16.59 \\
\hline 55 & 9_1_1 & 162.00 & 60.67 & 101.33 & 135.33 & 2.61 & 3.69 & 13.28 \\
\hline 56 & $9 \_1 \_2$ & 162.00 & 62.00 & 100.00 & 132.00 & 2.56 & 3.67 & 15.40 \\
\hline 57 & 15_1_1 & 165.67 & 59.33 & 106.33 & 129.33 & 2.51 & 3.74 & 13.74 \\
\hline 58 & 15_1_2 & 162.67 & 59.67 & 103.00 & 129.33 & 2.61 & 3.98 & 11.85 \\
\hline 59 & $259 \_1$ & 162.67 & 70.00 & 92.67 & 119.67 & 2.59 & 3.81 & 11.86 \\
\hline 60 & $259 \_2$ & 166.33 & 70.33 & 96.00 & 127.00 & 2.65 & 3.68 & 13.03 \\
\hline 61 & 10_1 & 157.67 & 65.33 & 92.33 & 142.33 & 2.55 & 3.46 & 14.45 \\
\hline 62 & 10_2 & 160.33 & 60.00 & 100.33 & 142.00 & 2.52 & 3.53 & 12.97 \\
\hline 63 & 17_2_1 & 158.00 & 72.00 & 86.00 & 140.33 & 2.66 & 3.95 & 12.57 \\
\hline 64 & 17_2_2 & 162.00 & 72.00 & 90.00 & 143.67 & 2.67 & 4.15 & 13.82 \\
\hline 65 & $20 \_1$ & 167.67 & 63.00 & 104.67 & 153.00 & 2.52 & 3.74 & 16.59 \\
\hline 66 & $20 \_2$ & 170.33 & 64.67 & 105.67 & 156.67 & 2.60 & 4.02 & 15.75 \\
\hline 67 & $23 \_1$ & 156.33 & 53.00 & 103.33 & 151.67 & 2.49 & 3.53 & 17.37 \\
\hline 68 & 23_2 & 155.00 & 51.33 & 103.67 & 153.00 & 2.39 & 3.46 & 17.37 \\
\hline 69 & 63_1 & 165.67 & 67.33 & 98.33 & 155.67 & 2.54 & 3.73 & 15.59 \\
\hline 70 & 63_2 & 167.33 & 71.67 & 95.67 & 158.00 & 2.57 & 3.62 & 15.15 \\
\hline 71 & 90_1 & 153.67 & 66.67 & 87.00 & 112.33 & 2.80 & 3.77 & 13.15 \\
\hline 72 & $90 \_2$ & 152.67 & 63.00 & 89.67 & 110.67 & 2.76 & 3.94 & 13.86 \\
\hline 73 & 124_1 & 146.33 & 45.67 & 100.67 & 129.33 & 2.61 & 3.45 & 15.89 \\
\hline 74 & $124 \_2$ & 141.33 & 45.67 & 95.67 & 133.67 & 2.53 & 3.65 & 14.74 \\
\hline 75 & 126_1 & 147.00 & 46.67 & 100.33 & 147.00 & 2.48 & 3.77 & 15.83 \\
\hline 76 & $126 \_2$ & 149.00 & 47.33 & 101.67 & 146.33 & 2.56 & 3.61 & 15.28 \\
\hline 77 & 169_1 & 158.00 & 77.33 & 80.67 & 122.33 & 2.59 & 3.49 & 12.01 \\
\hline 78 & $169 \_2$ & 158.00 & 74.67 & 83.33 & 126.33 & 2.55 & 3.64 & 12.08 \\
\hline 79 & $55 \_2 \_1$ & 164.67 & 66.33 & 98.33 & 133.33 & 2.75 & 3.34 & 14.26 \\
\hline 80 & $55 \_2 \_2$ & 168.00 & 67.33 & 100.67 & 127.33 & 2.65 & 3.47 & 14.85 \\
\hline 81 & Intr. No. 153515 & 143.67 & 56.00 & 87.67 & 125.67 & 2.43 & 3.55 & 13.25 \\
\hline 82 & Intr. No. 158071 & 143.67 & 60.33 & 83.33 & 128.67 & 2.70 & 3.61 & 11.68 \\
\hline 83 & Giza25 & 143.67 & 57.67 & 86.00 & 127.00 & 2.72 & 3.72 & 12.97 \\
\hline 84 & Giza32 & 141.67 & 50.00 & 91.67 & 129.00 & 2.50 & 3.58 & 12.67 \\
\hline 85 & Shandweil3 & 128.00 & 31.67 & 96.33 & 107.33 & 3.01 & 4.04 & 13.54 \\
\hline \multirow[t]{3}{*}{86} & Toshkal & 132.00 & 28.67 & 103.33 & 108.67 & 3.17 & 4.31 & 13.72 \\
\hline & RLSD $_{.05}$ & 6.68 & 4.76 & 7.97 & 14.38 & 0.104 & 0.266 & 1.89 \\
\hline & $\operatorname{RLSD}_{.01}$ & 8.64 & 6.16 & 10.40 & 18.78 & 0.135 & 0.347 & 2.47 \\
\hline
\end{tabular}

RLSD.05 and RLSD.01, Revised L.S.D at 0.05 and 0.01 , respectively. 
TABLE 3. Means of studied traits for the 86 sesame genotypes in 2016 season.

\begin{tabular}{|c|c|c|c|c|c|c|c|c|}
\hline $\begin{array}{l}\text { Genotype } \\
\text { No. }\end{array}$ & Code & $\begin{array}{l}\text { Plant } \\
\text { height } \\
(\mathrm{cm})\end{array}$ & $\begin{array}{c}\text { First } \\
\text { capsule } \\
\text { height } \\
(\mathrm{cm})\end{array}$ & $\begin{array}{c}\text { Fruiting } \\
\text { zone } \\
\text { length } \\
\text { (cm) }\end{array}$ & $\begin{array}{l}\text { Number } \\
\text { of capsules } \\
\text { per plant }\end{array}$ & $\begin{array}{c}\text { Capsule } \\
\text { length } \\
\text { (cm) }\end{array}$ & $\begin{array}{c}\text { Thousand } \\
\text { seed } \\
\text { weight } \\
\text { (g) }\end{array}$ & $\begin{array}{c}\text { Seed } \\
\text { yield } \\
(\mathrm{g})\end{array}$ \\
\hline 1 & 1_1_1 & 175.33 & 65.67 & 109.67 & 146.00 & 2.39 & 3.54 & 13.00 \\
\hline 2 & 1_1_2 & 178.33 & 67.67 & 110.67 & 145.67 & 2.50 & 3.50 & 15.28 \\
\hline 3 & 5_1_1 & 178.00 & 74.33 & 103.67 & 148.00 & 2.47 & 3.61 & 16.34 \\
\hline 4 & $5 \_1 \_2$ & 173.67 & 74.33 & 99.33 & 148.00 & 2.63 & 3.47 & 15.87 \\
\hline 5 & 9_1_1 & 174.67 & 76.33 & 98.33 & 153.67 & 2.65 & 3.86 & 16.78 \\
\hline 6 & 9_1_2 & 173.33 & 75.00 & 98.33 & 158.00 & 2.59 & 3.75 & 18.94 \\
\hline 7 & 9_2_1 & 167.67 & 79.67 & 88.00 & 141.33 & 2.52 & 4.22 & 13.17 \\
\hline 8 & 9_2_2 & 168.33 & 80.00 & 88.33 & 138.67 & 2.52 & 3.87 & 14.98 \\
\hline 9 & 10_1 & 168.00 & 86.00 & 82.00 & 140.33 & 2.53 & 3.76 & 15.62 \\
\hline 10 & $10 \_2$ & 169.67 & 84.00 & 85.67 & 132.33 & 2.57 & 3.88 & 16.11 \\
\hline 11 & $11 \_2 \_1$ & 167.33 & 73.67 & 93.67 & 161.00 & 2.49 & 3.83 & 16.58 \\
\hline 12 & $11 \_2 \_2$ & 166.00 & 75.00 & 91.00 & 162.67 & 2.46 & 4.23 & 17.58 \\
\hline 13 & $266 \_1$ & 156.67 & 64.33 & 92.33 & 152.33 & 2.37 & 3.87 & 15.82 \\
\hline 14 & $266 \_2$ & 156.67 & 62.67 & 94.00 & 149.67 & 2.40 & 3.95 & 15.06 \\
\hline 15 & 216_1 & 159.67 & 61.67 & 98.00 & 146.00 & 2.45 & 3.92 & 15.34 \\
\hline 16 & $216 \_2$ & 160.67 & 64.33 & 96.33 & 148.33 & 2.42 & 3.71 & 15.63 \\
\hline 17 & 257_1 & 156.33 & 56.67 & 99.67 & 138.67 & 2.40 & 3.52 & 11.79 \\
\hline 18 & $257 \_2$ & 162.00 & 58.67 & 103.33 & 128.67 & 2.59 & 3.66 & 12.37 \\
\hline 19 & $44 \_1$ & 170.67 & 64.67 & 106.00 & 126.00 & 2.49 & 3.37 & 12.91 \\
\hline 20 & 44_2 & 171.67 & 70.67 & 101.00 & 123.67 & 2.41 & 3.23 & 13.09 \\
\hline 21 & 34_2_1 & 171.33 & 72.33 & 99.00 & 137.00 & 2.65 & 3.19 & 16.95 \\
\hline 22 & 34_2_2 & 173.67 & 70.67 & 103.00 & 139.33 & 2.69 & 3.13 & 16.35 \\
\hline 23 & 59_1_1 & 158.33 & 46.00 & 112.33 & 139.00 & 2.58 & 4.01 & 14.49 \\
\hline 24 & 59_1_2 & 158.67 & 52.00 & 106.67 & 139.67 & 2.65 & 3.51 & 14.67 \\
\hline 25 & 110_12_1 & 155.00 & 60.67 & 94.33 & 107.33 & 2.62 & 3.61 & 12.40 \\
\hline 26 & 110_12_2 & 155.33 & 59.00 & 96.33 & 129.33 & 2.77 & 3.60 & 12.03 \\
\hline 27 & 58_3_1 & 148.00 & 60.67 & 87.33 & 138.00 & 2.57 & 3.11 & 14.13 \\
\hline 28 & $58 \_3 \_2$ & 153.67 & 56.33 & 97.33 & 141.33 & 2.70 & 3.34 & 14.25 \\
\hline 29 & $58 \_1$ & 160.67 & 55.67 & 105.00 & 143.67 & 2.76 & 3.88 & 17.62 \\
\hline 30 & $58 \_2$ & 158.00 & 60.00 & 98.00 & 140.67 & 2.71 & 3.91 & 17.41 \\
\hline 31 & 167_1_1 & 154.33 & 40.67 & 113.67 & 122.00 & 2.46 & 3.46 & 11.78 \\
\hline 32 & 167_1_2 & 160.67 & 43.00 & 117.67 & 119.00 & 2.41 & 3.65 & 12.31 \\
\hline 33 & $148 \_1$ & 151.00 & 48.33 & 102.67 & 146.67 & 2.55 & 4.19 & 18.04 \\
\hline 34 & $148 \_2$ & 154.67 & 48.67 & 106.00 & 151.33 & 2.70 & 4.87 & 16.98 \\
\hline 35 & $65 \_1$ & 141.67 & 38.67 & 103.00 & 112.67 & 2.59 & 4.12 & 12.90 \\
\hline 36 & $65 \_2$ & 152.33 & 45.00 & 107.33 & 115.00 & 2.50 & 3.76 & 12.28 \\
\hline 37 & 76_1 & 167.33 & 79.00 & 88.33 & 144.67 & 2.47 & 3.84 & 14.71 \\
\hline 38 & $76 \_2$ & 166.33 & 83.67 & 82.67 & 142.33 & 2.44 & 3.73 & 15.79 \\
\hline 39 & $1 \_1$ & 155.67 & 54.67 & 101.00 & 153.00 & 2.56 & 2.99 & 17.53 \\
\hline 40 & $1 \_2$ & 159.00 & 53.00 & 106.00 & 155.67 & 2.55 & 3.43 & 17.12 \\
\hline 41 & 2_4_1 & 161.67 & 38.00 & 123.67 & 138.00 & 2.49 & 3.37 & 14.85 \\
\hline 42 & 2_4_2 & 159.67 & 44.33 & 115.33 & 138.00 & 2.51 & 3.48 & 13.03 \\
\hline 43 & 3_1 & 173.33 & 53.67 & 119.67 & 133.67 & 2.50 & 3.73 & 14.84 \\
\hline 44 & 32 & 170.67 & 46.67 & 124.00 & 134.67 & 2.49 & 3.79 & 14.48 \\
\hline
\end{tabular}


TABLE 3. Cont.

\begin{tabular}{|c|c|c|c|c|c|c|c|c|}
\hline $\begin{array}{l}\text { Genotype } \\
\text { No. }\end{array}$ & Code & $\begin{array}{c}\text { Plant } \\
\text { height } \\
(\mathrm{cm})\end{array}$ & $\begin{array}{c}\text { The first } \\
\text { capsule } \\
\text { heigh } \\
(\mathrm{cm})\end{array}$ & $\begin{array}{c}\text { Fruiting } \\
\text { zone } \\
\text { length } \\
\text { (cm) } \\
\end{array}$ & $\begin{array}{c}\text { Number } \\
\text { of capsules } \\
\text { per plant }\end{array}$ & $\begin{array}{c}\text { Capsule } \\
\text { length } \\
(\mathrm{cm})\end{array}$ & $\begin{array}{c}\text { Thousand } \\
\text { seed weight } \\
\text { (g) }\end{array}$ & $\begin{array}{c}\text { Seed } \\
\text { yield } \\
(\mathrm{g})\end{array}$ \\
\hline 45 & $58 \_1$ & 162.33 & 75.33 & 87.00 & 141.00 & 2.56 & 3.60 & 14.65 \\
\hline 46 & $58 \_2$ & 161.33 & 71.33 & 90.00 & 139.67 & 2.55 & 3.37 & 14.64 \\
\hline 47 & 125_1 & 150.00 & 39.67 & 110.33 & 126.33 & 2.53 & 3.46 & 11.13 \\
\hline 48 & $125 \_2$ & 155.00 & 45.33 & 109.67 & 125.67 & 2.53 & 3.58 & 12.35 \\
\hline 49 & $133 \_1$ & 170.00 & 82.00 & 88.00 & 154.00 & 2.44 & 3.19 & 17.65 \\
\hline 50 & $133 \_2$ & 167.67 & 81.33 & 86.33 & 155.33 & 2.49 & 3.52 & 16.54 \\
\hline 51 & $2 \_1 \_1$ & 160.67 & 59.67 & 101.00 & 121.67 & 2.45 & 3.34 & 11.21 \\
\hline 52 & 2_1_2 & 160.33 & 60.33 & 100.00 & 123.00 & 2.40 & 3.57 & 12.91 \\
\hline 53 & 11_1_1 & 163.33 & 68.00 & 95.33 & 149.33 & 2.57 & 3.91 & 16.85 \\
\hline 54 & 11_1_2 & 166.67 & 67.33 & 99.33 & 149.00 & 2.60 & 3.79 & 17.18 \\
\hline 55 & 9_1_1 & 162.33 & 60.67 & 101.67 & 137.67 & 2.59 & 3.70 & 14.33 \\
\hline 56 & 9_1_2 & 163.00 & 62.00 & 101.00 & 140.00 & 2.55 & 3.73 & 15.64 \\
\hline 57 & 15_1_1 & 164.33 & 59.33 & 105.00 & 126.33 & 2.54 & 3.75 & 14.55 \\
\hline 58 & 15_1_2 & 165.00 & 59.67 & 105.33 & 127.00 & 2.61 & 3.92 & 11.87 \\
\hline 59 & $259 \_1$ & 164.00 & 70.00 & 94.00 & 126.67 & 2.62 & 3.85 & 12.05 \\
\hline 60 & $259 \_2$ & 168.00 & 70.33 & 97.67 & 134.00 & 2.67 & 3.66 & 13.25 \\
\hline 61 & $10 \_1$ & 160.00 & 65.33 & 94.67 & 138.33 & 2.55 & 3.58 & 14.48 \\
\hline 62 & $10 \_2$ & 163.33 & 60.00 & 103.33 & 140.67 & 2.53 & 3.53 & 13.94 \\
\hline 63 & 17_2_1 & 159.00 & 72.00 & 87.00 & 147.33 & 2.69 & 3.94 & 12.92 \\
\hline 64 & 17_2_2 & 164.00 & 72.00 & 92.00 & 148.67 & 2.70 & 4.04 & 13.99 \\
\hline 65 & 20_1 & 168.67 & 63.00 & 105.67 & 155.00 & 2.55 & 3.76 & 16.93 \\
\hline 66 & $20 \_2$ & 171.00 & 64.67 & 106.33 & 158.67 & 2.60 & 4.02 & 16.35 \\
\hline 67 & $23 \_1$ & 157.33 & 53.00 & 104.33 & 150.33 & 2.47 & 3.58 & 17.96 \\
\hline 68 & $23 \_2$ & 155.33 & 51.33 & 104.00 & 150.67 & 2.41 & 3.46 & 18.35 \\
\hline 69 & $63 \_1$ & 167.67 & 67.33 & 100.33 & 161.00 & 2.57 & 3.75 & 15.88 \\
\hline 70 & $63 \_2$ & 164.33 & 71.67 & 92.67 & 163.67 & 2.59 & 3.62 & 15.98 \\
\hline 71 & 90_1 & 155.00 & 66.67 & 88.33 & 106.67 & 2.82 & 3.81 & 13.00 \\
\hline 72 & $90 \_2$ & 153.67 & 63.00 & 90.67 & 103.33 & 2.80 & 3.94 & 13.14 \\
\hline 73 & 124_1 & 148.33 & 45.67 & 102.67 & 130.67 & 2.62 & 3.53 & 15.98 \\
\hline 74 & $124 \_2$ & 143.33 & 45.67 & 97.67 & 131.33 & 2.56 & 3.70 & 15.56 \\
\hline 75 & $126 \_1$ & 148.67 & 46.67 & 102.00 & 150.00 & 2.49 & 3.80 & 15.55 \\
\hline 76 & $126 \_2$ & 152.33 & 47.33 & 105.00 & 148.33 & 2.53 & 3.62 & 16.75 \\
\hline 77 & 169_1 & 157.33 & 77.33 & 80.00 & 129.67 & 2.60 & 3.47 & 13.02 \\
\hline 78 & 169_2 & 160.33 & 74.67 & 85.67 & 127.67 & 2.58 & 3.63 & 12.38 \\
\hline 79 & 55_2_1 & 167.00 & 66.33 & 100.67 & 133.33 & 2.76 & 3.35 & 13.88 \\
\hline 80 & $55 \_2 \_2$ & 167.00 & 67.33 & 99.67 & 124.67 & 2.66 & 3.44 & 15.77 \\
\hline 81 & Intr. No. 153515 & 144.33 & 56.00 & 88.33 & 122.67 & 2.43 & 3.60 & 12.93 \\
\hline 82 & Intr. No. 158071 & 149.67 & 60.33 & 89.33 & 135.33 & 2.71 & 3.60 & 12.12 \\
\hline 83 & Giza25 & 145.67 & 57.67 & 88.00 & 129.67 & 2.73 & 3.75 & 13.67 \\
\hline 84 & Giza32 & 143.67 & 50.00 & 93.67 & 132.33 & 2.50 & 3.66 & 13.63 \\
\hline 85 & Shandweil3 & 128.67 & 31.67 & 97.00 & 111.00 & 3.05 & 4.12 & 14.63 \\
\hline \multirow[t]{3}{*}{86} & Toshka1 & 128.00 & 28.67 & 99.33 & 113.67 & 3.18 & 4.15 & 14.78 \\
\hline & $\operatorname{RLSD}_{.05}$ & 8.47 & 4.83 & 9.51 & 11.48 & 0.079 & 0.264 & 1.92 \\
\hline & $\operatorname{RLSD}_{.01}$ & 11.05 & 6.25 & 12.84 & 14.99 & 0.102 & 0.344 & 2.51 \\
\hline
\end{tabular}

RLSD.05, RLSD.01, Revised L.S.D at 0.05 and 0.01 , respectively 

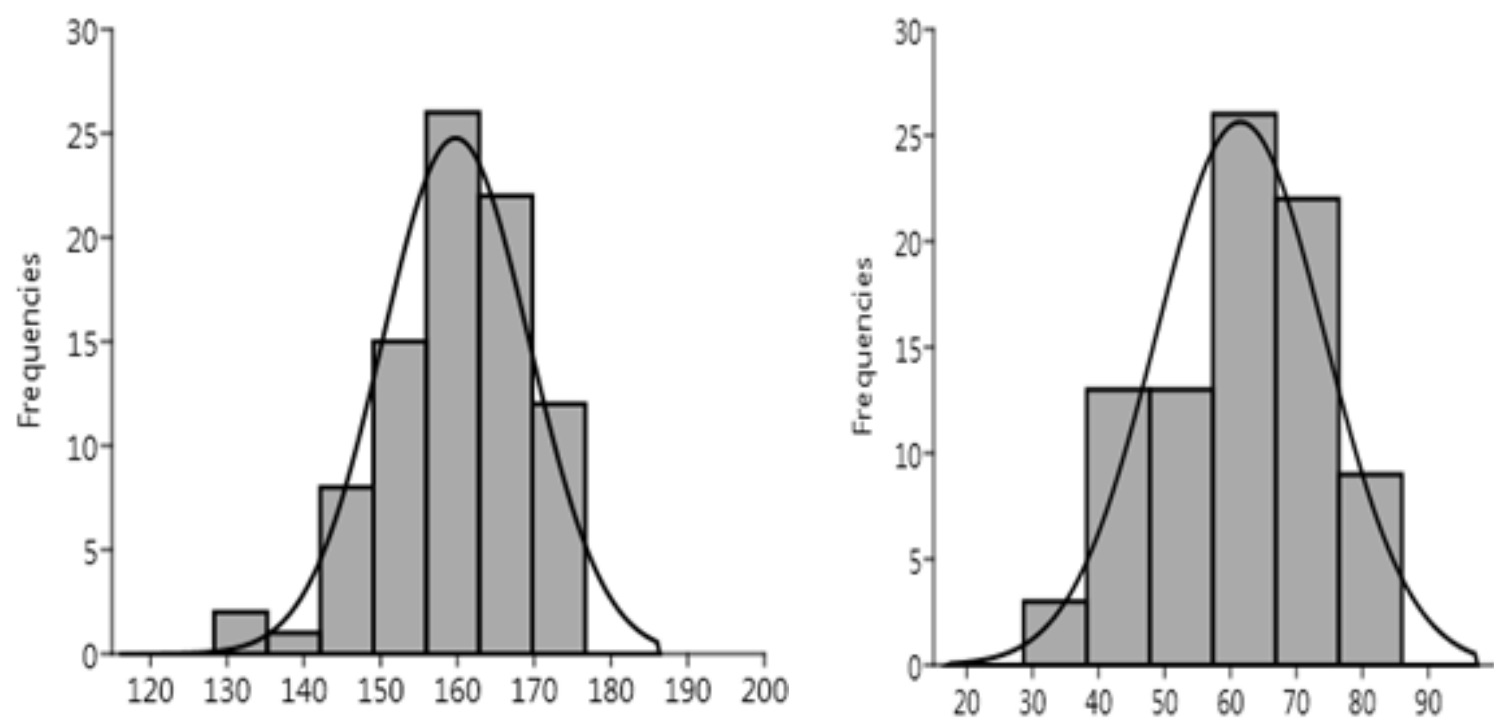

Fig. 1. Frequency distribution of sesame lines means over two years for (on the left) the plant height (cm) and (on the right) the first capsule height $(\mathrm{cm})$.
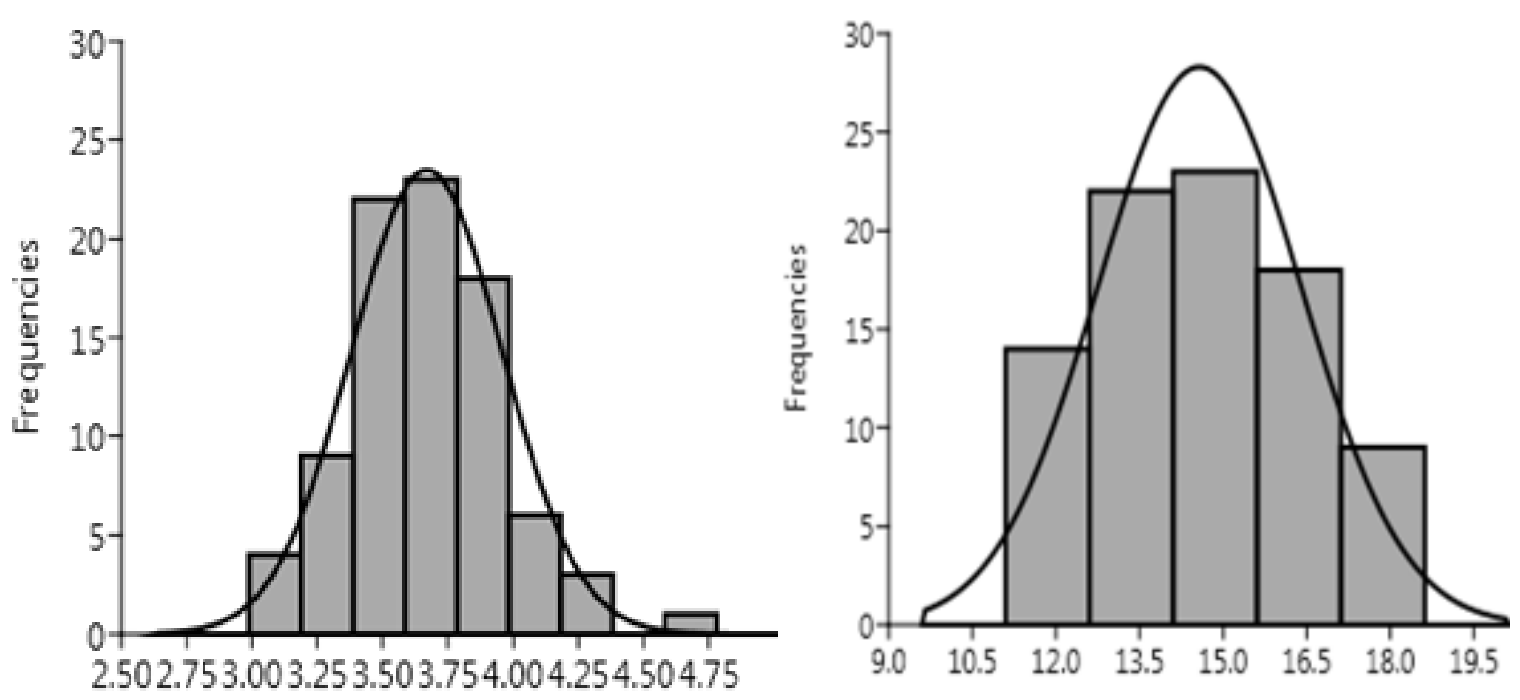

Fig. 2. Frequency distribution of sesame lines means over two years for (on the left) the thousand seeds weight (g) and (on the right) the seed yield per plant (g).

The heritability estimated in broad sense solo is not reliable, because these estimations values are might be altered with any changes of environment and plant materials. Patil \& Lokesha (2018) said that using high broad sense heritability coupled with higher genetic advance is more preferred and helpful in prediction gain under the selection.

The data in the Table 1 represent a low genetic advance for the capsule length (0.25 and 0.26), the thousand seed weight $(0.56$ and 0.54$)$ and the seed yield per plant (3.25 and 3.57) coupled with high heritability estimations in the first and second seasons, respectively. Obtained results due to the capsule length, the thousand seed weight and the seed yield per plant are controlled by multi genes, complex nature and much affected by the environment. Moderate values of genetic advance were observed for plant height (18.49) in both seasons and (17.52 and 16.94) for the fruiting zone length in the first and second seasons, respectively coupled with high heritability. These results refer to the presence of additive and non-additive genes. While, the high heritability coupled with high genetic advance values were recorded for the first capsule height (26.05 and 25.85) and the capsules number per plant (22.55 and 26.11) in the first and second seasons, respectively. These traits might be 
controlled by the additive gene action mostly which is very useful for selection efficiency. Similar results were reported by El Soury et al. (2016) and Patil \& Lokesha (2018).

The genetic advance represented in the Table 1 as a percent of means (GAM) was moderate (10-20\%) for the plant height, the fruiting zone length, the capsules number per plant, the capsule length and the thousand seed weight in both seasons. The GAM of first capsule height (42.32 and $42.08 \%)$ and the seed yield per plant $(22.61$ and $24.19 \%$ ) were high percentages $(>20 \%)$ in the first and second season respectively, coupled with high heritability and low differences between the PCV and the GCV (Fig. 3). That is meaning simple selection based on the phenotypes after a cycle of selection at $5 \%$ intensity for the first capsule height and the seed yield per plant would be effective. Hika et al. (2015) observed the higher GAM estimates (>30\%) for the seed yield per ha, biomass yield per ha and the harvest index. In the current study, the genetic variability estimations of studied traits revealed that the first capsule height, the seed yield per plant, fruiting zone length and the capsules number per plant was the highest GAM coupled with high heritability. So, this phenomenon suggests that the additive gene action model controls these traits and the mass selection would be effective for the sesame productivity improvement.
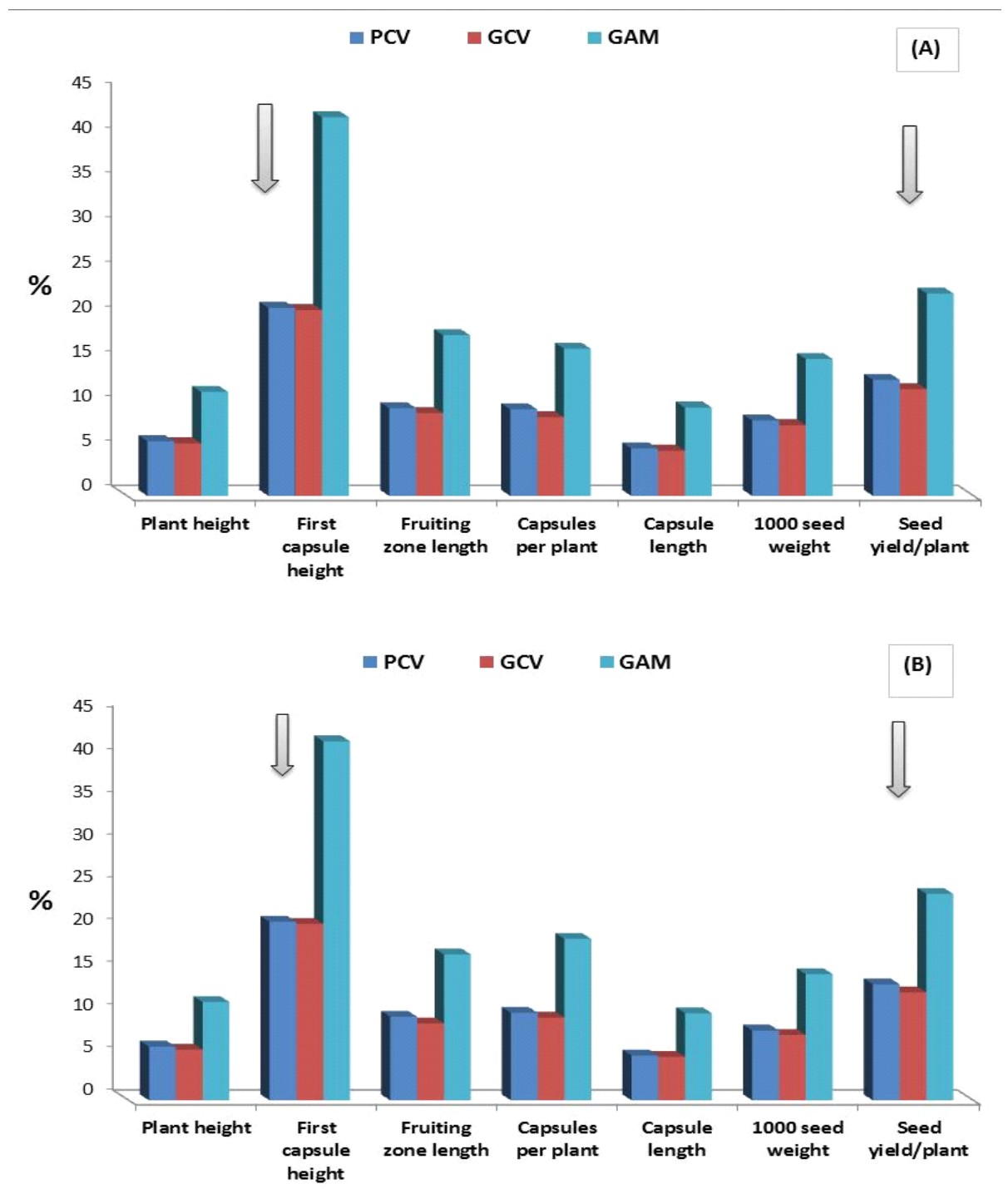

Fig. 3. Estimations of the PCV, the GCV and the genetic advance as percent of means in the first season (A) and second season (B). 


\section{Conclusion}

In the current study, the genetic variability estimations of studied traits revealed that the first capsule height, the seed yield per plant, fruiting zone length and the capsules number per plant was the highest GAM coupled with high heritability. So, this phenomenon suggests that the additive gene action model controls these traits and the mass selection would be effective for the sesame productivity improvement.

\section{References}

Abate, M., Mekbib, F., Ayana, A. and Nigussie, M. (2015) Genetic variability and association of traits in midaltitude sesame (Sesamum indicum L.) germplasm of Ethiopia. Amer. J. Exp. Agric. 9(3), $1-14$.

Al-Jibouri, H.A., Miller, P.A. and Robinson, H.F. (1958) Genotypic and environmental variance and covariance in Upland cotton cross of interspecific origin. Agron. J. 50, 633-636.

Aristya, V.E., Taryono, T. and Wulandari, R.A. (2017) Genetic variability, standardized multiple linear regression and principal component analysis to determine some important sesame yield components. Agri. Vita. J. Agric. Sci. 39(1), 83-90.

Ashri, A. (1998) Sesame breeding. Plant Breeding Review, 16, 179-228.

Aye, M., Khaing, T.T. and Hom, N.H. (2018) Morphological characterization and genetic divergence in Myanmar Sesame (Sesamum indicum L.) germplasm. Int. J. Adv. Res. 6(4), 297-307.

Begum, T. and Dasgupta, T. (2014) Genetic variability estimates in induced population of sesame (Sesamum indicum L.). Indian J. Agric. Sci. 84, 551-554.

Begum, T., Iqbal, A. and Dasgupta, T. (2017) Genetic variability and divergence among genotypes of Sesame (Sesamum inducum L.). Bangladesh $J$. Bot. 46(3), 955-962.

Bharathi, D., Thirumalarao, V., Chandramohan, Y., Bhadru, D. and Venkanna, V. (2014) Genetic variability studies in sesame (Sesamum indicum L.). Int. J. Appl. Biol. Pharma. Technol. 5(4), 31-33.

Boureima, S., Diouf, M., Amoukou, A.I. and Van
Damme, P. (2016) Screening for sources of tolerance to drought in sesame induced mutants: Assessment of indirect selection criteria for seed yield. Int. J. Pure App. Bio Sci. 4(1), 45-60.

Burton, G.W. (1952) Quantitative inheritance in grasses. $6^{\text {th }}$ International Grassland Cong. Proc. 1, 227-238.

El Soury, H.F., El Bashir, G. and Ginaro, M.K. (2016) Phenotypic and genotypic coefficients of variation and other growth attributes in sesame genotype under rain-fed conditions. Adv. Agric. Agric. Sci. 2(3), 79-84.

FAOSTAT (2016) Food and agriculture organization of the United Nations, http://faostat.fao.org.

Gidey, Y.T., Kebede, S.A. and Gashawbeza, G.T. (2012) Extent and pattern of genetic diversity for morpho-agronomic traits in Ethiopian Sesame Landraces (Sesamum indicum L.). Asian J. Agric. Res. 6, 118-128.

Hamza, M. and Abd El-Salam, R.M. (2015) Optimum planting date for three sesame cultivars growing under sandy soil conditions in Egypt. J. Agric. \& Environ. Sci. 15(5), 868-877.

Hassan, M.A.M (2013) Studies on Egyptian sesame seeds (Sesamum indicum L.) and its products. 3. Effect of roasting process on gross chemical composition, functional properties, antioxidative components and some minerals of defatted sesame seeds meal (Sesamum indicum L.). World J. Dairy \& Food Sci. 8(1), 51-57.

Hika, G., Geleta, N. and Jaleta, Z. (2015) Genetic variability, heritability and genetic advance for the phenotypic traits in Sesame (Sesamum indicum L.) populations from Ethiopia. Sci. Technol. Arts Res. J. 4(1), 20-26.

Khan, M.A., Mirza, M.Y., Akmal, M., Ali, N. and Khan, I. (2007) Genetic parameters and their implications for yield improvement in Sesame. Sarhad J. Agric. 23(3), 623-627.

Mahdy, E.E., Bakheit, B.R., Motawea, M.M. and Bedawy, I.M. (2005a) Pedigree selection for resistance to Sclerotium bataticola in three sesame populations. Assuit J. Agric.Sci. 63(1), 57-72.

Mahdy, E.E., Bakheit, B.R., Motawea, M.M. and 
Bedawy, I.M. (2005 ) Pedigree selection for resistance to Fusarium oxysporum in three sesame populations. Assuit J. Agric.Sci. 63(1), 141-157.

Patil, M.K. and Lokesha, R. (2018) Estimation of genetic variability, heritability, genetic advance, correlations and path analysis in advanced mutant breeding lines of sesame (Sesamum indicum L.). J. Pharmacog. Nat. Prod. An open Access Journal, 4(1), 100-151.

Petersen, R.G. (1985) "Design and Analysis of Experiments". Marcel Dekker, Inc. New York. 436pp.

Sabiel, S.A.I., Ismail, M.I., Abdalla, E.A. and Osman, A.A. (2015) Genetic variation in sesame genotypes (Sesamum indicum L.) grown in the semi-arid zone of Sudan. SABRAO J. Breed. Genet. 47(3), 214-220.

Saha, S., Begum, T. and Dasgupta, T. (2012) Analysis of genotypic diversity in sesame based on morphological and agronomic traits. In: Proc. Tropentag 2012: International conference on "Resilience of agricultural systems against crises" held at Göttingen, Germany during September 1921.

SAS Institute (2008) The SAS system for Windows, release 9.2. SAS Institute, Cary, N.C. USA.
Saxena, K. and Bisen, R. (2017) Genetic variability, heritability and genetic advance for the phenotypic traits in sesame (Sesamum indicum L.). Int. J. Pure App. Biosci. 5(2), 1126-1131.

Shekhawat, R.S., Rajput, S.S., Meena, S.K. and Singh, B. (2013) Variation and character association in seed yield and related traits in sesame (Sesamum indicum L.). Indian Research J. Genet. Biotechnol. 5(3), 186-193.

Spandana, B., Anuradha, G., Sivaraj, N. and Sivarama, K. (2011) Determination of genetic variation in Indian sesame (Sesamum indicum L.) genotypes for agro-morphological traits. J. Res. Agric.Sci. 7, 88-99.

Weiss, E.A. (2000) "Oilseed Crops", $2^{\text {nd }}$ ed, Ch., 5, pp. 131-164. Blackwell Science, Oxford.

Yol, E. and Uzun, B. (2012) Geographical patterns of sesame (Sesamum indicum L.) accessions grown under Mediterranean environmental conditions, and establishment of a core collection. Crop Sci. 52, 2206-2214 


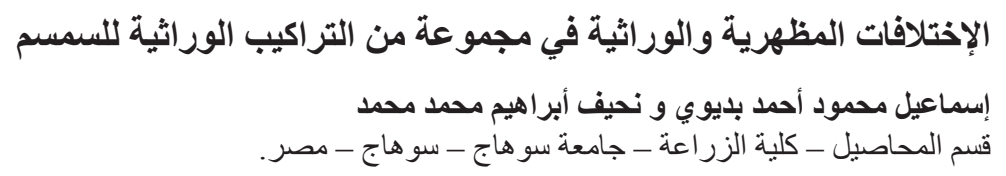

اجريت هذه الدراسة خلال الموسم الصيفى لعامى 2015 , 2016 لدر اسة الإختالافات المظهرية و الور اثية

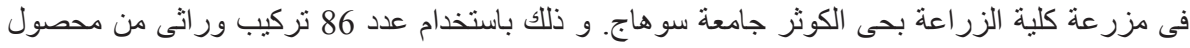

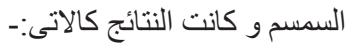

اظهرت نتائج التحليل الاحصائى وجود اختلافات معنوية عند مستوى معنوية 1\% بين التر اكيب الور اثثية لجميع الصفات الددروسة فى كلا الموسمين.

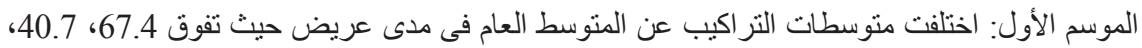

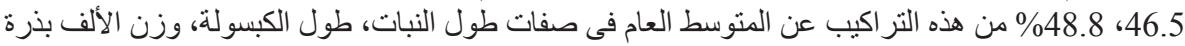

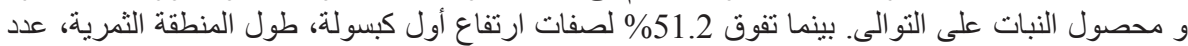

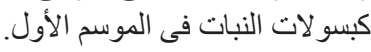

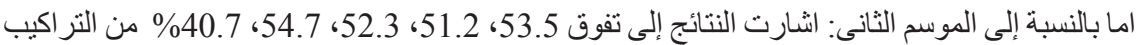

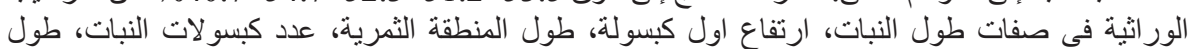
الكبسولة على التولى، 48.8\% فى صفتى وزن الألف بذرة و محصول النبات النقات

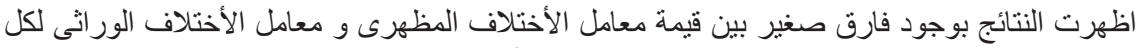

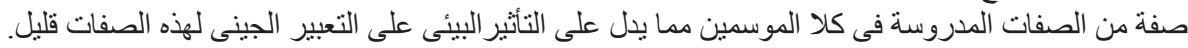

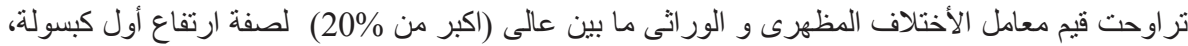

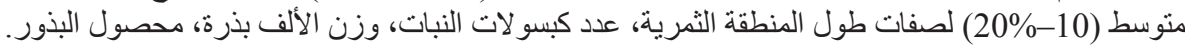
على الجانب الأخر وجدت قيم منخفضة لمعامل الإختلاف الور اثى و المظهرى (اقل من الثر 10\%) لصفات طول

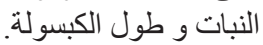

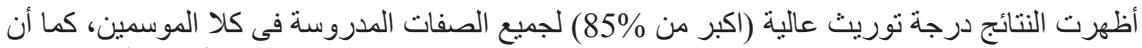

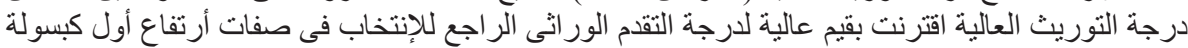

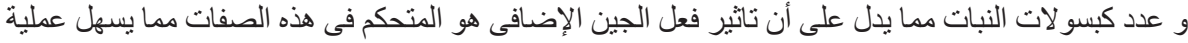

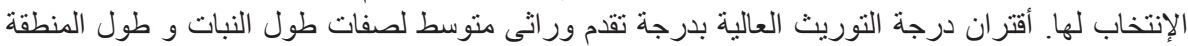

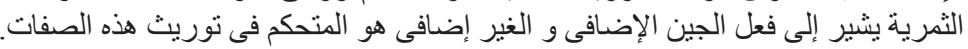

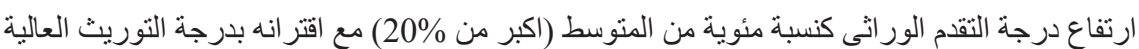

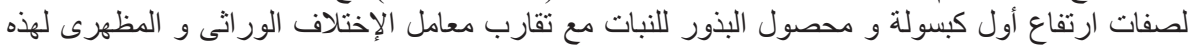

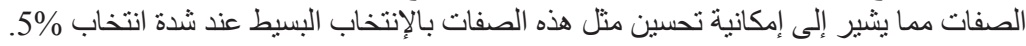

